\title{
Modeling of an Electric Vehicle Thermal Management System in MATLAB/Simulink
}

\section{Tibor Kiss, Jason Lustbader, and Daniel Leighton}

National Renewable Energy Laboratory

CITATION: Kiss, T., Lustbader, J., and Leighton, D., "Modeling of an Electric Vehicle Thermal Management System in MATLAB/

Simulink," SAE Technical Paper 2015-01-1708, 2015, doi:10.4271/2015-01-1708.

Copyright (C) 2015 SAE International

\begin{abstract}
Electric vehicles (EVs) need highly optimized thermal management systems to improve range. Climate control can reduce vehicle efficiency and range by more than $50 \%$. Due to the relative shortage of waste heat, heating the passenger cabin in EVs is difficult. Cabin cooling can take a high portion of the energy available in the battery. Compared to internal combustion engine-driven vehicles, different heating methods and more efficient cooling methods are needed, which can make EV thermal management systems more complex. More complex systems typically allow various alternative modes of operation that can be selected based on driving and ambient conditions. A good system simulation tool can greatly reduce the time and expense for developing these complex systems. A simulation model should also be able to efficiently co-simulate with vehicle simulation programs, and should be applicable for evaluating various control algorithms. The MATLAB/Simulink dynamic system simulation environment, widely used in the automotive industry, effectively meets these criteria.
\end{abstract}

To model the full EV thermal management system, the National Renewable Energy Laboratory's air-conditioning model now incorporates liquid-coolant system components. In the full system model, lookup tables were used to characterize the components' performance. Predicted data obtained with the system simulation model were compared against experimental data. An agreement within $5 \%$ for most of the system parameters was achieved. The validated system model was then used to determine which of two possible locations for the power electronics and electric motor in the system is better for quick cabin heating starting from cold soak.

\section{Introduction}

With improving vehicle efficiency, thermal systems are increasingly important for effective and efficient heavy- and light-duty vehicle design. Developing flexible and cost-effective tools to understand vehicle thermal trade-offs at the system level is critical to designing advanced electrified traction drive systems and their associated thermal controls.
When operating, the air conditioning (A/C) system is the largest auxiliary load on a conventional vehicle. $\mathrm{A} / \mathrm{C}$ loads account for more than $5 \%$ of the fuel used annually for light-duty vehicles in the United States [1]. Climate control loads can have an even larger impact on hybrid electric vehicle, plug-in hybrid electric vehicle, and all-electric vehicle (EV) performance. Hybrid EVs have $22 \%$ lower fuel economy with the $\mathrm{A} / \mathrm{C}$ on [2]. For all-electric vehicles, the effect of the climate control system can be even more severe. Due to the relative shortage of waste heat, heating the passenger cabin in EVs is difficult. Cooling the cabin can take a high portion of the energy available in the battery, significantly reducing vehicle efficiency and range. Mitsubishi reports that the range of the $\mathrm{i}-\mathrm{MiEV}$ can be reduced by as much as $68 \%$ for heating and $46 \%$ for cooling on the Japan 10-15 cycle []ㅡ. The Advanced Powertrain Research Facility at Argonne National Laboratory has reported 59.3\% and $53.7 \%$ reductions in range due to maximum heating and maximum cooling, respectively, for the Ford Focus EV operating on the Urban Dynamometer Driving Schedule cycle [4]. In addition to these climate control impacts, electric-drive vehicles (EDVs) may have additional cooling requirements for the electric traction drive system components, including batteries, power electronics, and electric machines.

Therefore, compared to internal combustion engine-driven vehicles, different heating methods and more efficient cooling methods are needed for EVs. These methods often involve running the $\mathrm{A} / \mathrm{C}$ system in heat pump mode for sufficient heating of the cabin. In some advanced concepts, the traditional liquid coolant-based thermal management is supplemented with the refrigerant-based cooling system, which can make the thermal management system more complex. When developing a thermal management system for an internal combustion engine vehicle, it has traditionally been sufficient to run models of the $\mathrm{A} / \mathrm{C}$ system and the liquid coolant-based cooling system separately. For advanced vehicles, especially for hybrid and all-electric vehicles, the interconnectedness of the thermal systems requires a more integrated simulation approach.

The more complex thermal management systems of advanced vehicles typically allow for various alternative modes of operation that can be selected based on driving and ambient conditions. Investigating a number of system alternatives and determining the 
best ranges for the various operating modes with experimental methods can be very time consuming. A good system simulation tool can greatly reduce the time and expense spent on the development of these complex systems. A simulation model should also be able to efficiently co-simulate with vehicle simulation programs and should be well applicable for evaluating various control algorithms. The MATLAB/Simulink dynamic system simulation environment is well suited for such system simulation models because it is widely used in the automotive industry and effectively meets these criteria.

\section{Applied Modeling Methods}

To meet the needs of advanced vehicle thermal system simulation, the National Renewable Energy Laboratory (NREL) is building on previously developed Simulink A/C models, adding liquid coolant loops to enable integrated system simulation. Simulink is a common engineering platform that allows for co-simulation with Autonomie [5]. NREL previously developed CoolSim, an A/C system simulation modeling framework in MATLAB/Simulink and validated its performance against test bench data. To match the wide range of $A / C$ modeling needs, NREL developed models with three different levels of detail: the Fully-Detailed, Quasi-Transient, and MappedComponent models, illustrated in Figure 1.

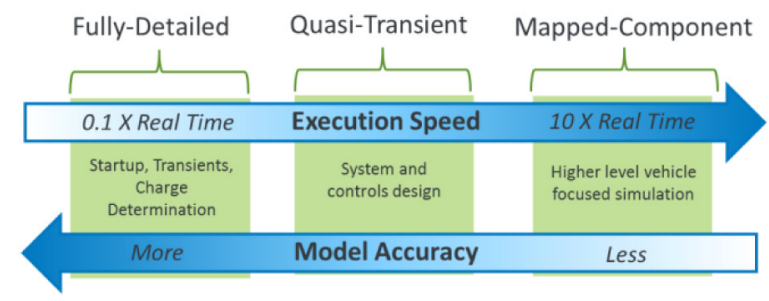

Figure 1. Previously developed A/C system model versions

In the trade-off of speed and accuracy, the three models occupy very different parts of the scale and meet a wide range of modeling needs. The Fully-Detailed model captures the detailed system transient behavior but runs at 0.1 real-time speed [ 6 ]. The Quasi-Transient and Mapped-Component models are progressively more simplified while trying to maintain accuracy and run at real-time speed and greater than 10 times real-time speed, respectively [7]. The goal of these new model versions was to provide faster simulation tools for less detailed, vehicle-focused drive-cycle-based evaluation of A/C systems. For steady-state conditions, the Quasi-Transient model provides essentially the same accuracy as the Fully-Detailed model. The Mapped-Component model does lose some accuracy in steadystate conditions. For the SC03 cycle, the averaged results of power and heat exchange rates obtained with the Quasi-Transient model are within $3 \%$ of the results of the Fully-Detailed model. The MappedComponent model results are within $15 \%$ of the results of the Fully-Detailed model. Short transients, such as those occurring during compressor cycling, produce the most deviation from the Fully-Detailed model for both simplified models. Conversion from one $\mathrm{A} / \mathrm{C}$ system model approach to another of the three models is relatively simple. This allows a new system model to be developed in a faster version before the results are refined using a slower, more detailed solution method as needed.

As outlined in the Introduction, there is a need for full thermal system simulation due to the interconnectedness of the refrigerant and liquid coolant circuits used in advanced thermal management systems, especially for EVs. Therefore, NREL's refrigerant circuit simulation model was extended with a liquid-coolant circuit simulation capability. The Quasi-Transient and the Mapped-Component model versions of the $\mathrm{A} / \mathrm{C}$ system simulation were used as the basis of the extension because they are the best fit in terms of model execution speed and accuracy. The new model was applied to an experimental advanced all-electric vehicle thermal management system.

Comparison of simulated results to measured data validated this particular system model and also the modeling methodology in general.

\section{Modeling the Liquid Coolant-Based System}

Solving the single-phase liquid coolant circuit model requires determining the coolant flow through the various branches in the system and the heat transfer of the components. These two aspects of the modeling will be discussed in the next two subsections

\section{Determining the Coolant Flow Rates throughout the System}

Some important processes in the liquid coolant part of the thermal management system take place over minutes and longer time periods. Therefore, it is important that the simulation model is able to run at near real-time speed. The two-phase refrigerant circuit model is already difficult to solve at such speed. It is therefore important that the liquid coolant simulation run as fast as possible on its own, so that together, the full system model has adequate speed. Pressure wave line dynamics are not important in the liquid coolant flow; therefore, an incompressible flow formulation was selected. This allows for a larger simulation time step. The Quasi-Transient and the Mapped-

Component refrigerant circuit simulations can also use this larger time step. In Simulink, it is difficult to have two parts of the model run at different simulation time steps; therefore, it makes sense to have the two model formulations run optimally with the same time-step.

During the development of a thermal system, various options for system design and operating modes may be investigated. Therefore, providing model flexibility is important. To achieve this coolant circuit flexibility, the liquid coolant fluid of the thermal system was modeled as a generic fluid network with loops and branches in which the flow rates are determined by the pressure changes (losses and rises) through various components (e.g., transport lines, heat exchangers, valves, pump) in the system (Figure 2).

To solve for the flows in a complex fluid network, two main sets of equations are considered: nodal continuity and loop equations. One continuity equation is written for each node, which states that the sum of the incoming flow rates should equal zero. For each fluid loop, an equation is written that states that the sum of pressure changes in each branch around the fluid loop should equal zero. These equations are analogous to the Kirchhoff laws for electric circuit theory, where they are written for the total incoming current in the nodes and for the sum of electric potential drop around conductor loops. The unknowns are the flow rates in the branches. To have the right number of equations for calculating the branch flow rates, the loops for which the loop equations are written need to be correctly selected. The nodal continuity equations provide $n_{n}-1$ equations, because one of these equations is a dependent equation, that is, it can be expressed as 
a linear combination of all the other nodal continuity equations. The number of unknowns are the number of branches, $n_{b}$. Therefore, the number of loops, $n_{l}$, has to be

$$
n_{l}=n_{b}-\left(n_{n}-1\right)=n_{b}-n_{n}+1
$$

It also important that the loop equations are independent or else the number of independent equations is not sufficient. One way to achieve this is to be sure each loop has at least one branch that belongs to that one loop only. There is still some freedom in how to identify the loops, and it makes sense to identify the loops used by the simulation model such that these are actual loops that the system reduces to in different modes of operation.

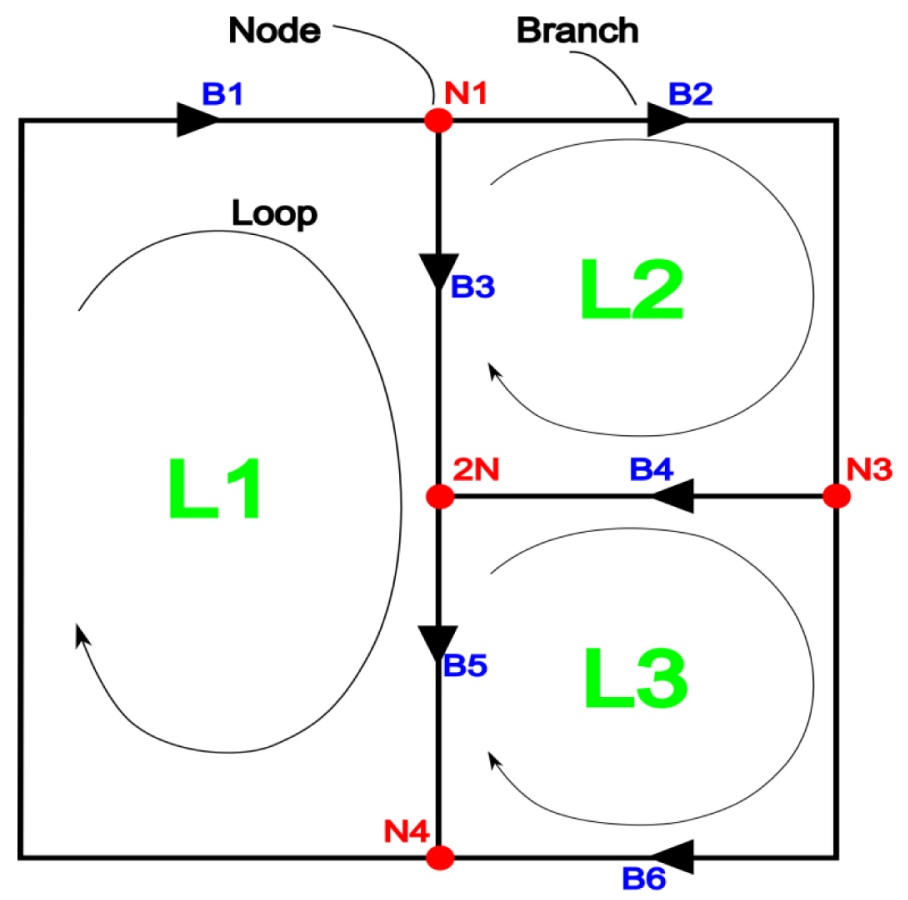

Figure 2. Fluid network example. Green font 'L': loops; Red font 'N': nodes; Blue font ' $\mathrm{B}$ ': branches

Once a valid set of independent flow loops is defined, the flow rates in the branches can be written as a superimposition of the flow rates in the loops. Note that with this method, the nodal continuity equations are automatically satisfied, and the number of unknowns in the system is reduced from the number of branches to the number of loops. The number of equations equals the number of unknowns, and the system can be solved.

The numerical process can be summarized as follows: assume a flow rate for each loop, calculate the branch flow rates from the loop flow rates with consideration of which loops each branch is a part, and then calculate the loop pressure drops which are the total of the branch pressure drops around each loop. If these loop pressure drops are not all zero within a small error, then iterate on the loop flow rates until they are all zero. This process is summarized in Figure 3 using the notation from Figure 2.

The iteration can be done to convergence within one simulation time-step; therefore, accurate flow rates can be obtained in each time step. To increase the execution speed, however, only the first iteration of this flow rate calculation process is executed at each time step. If boundary conditions on the system change slowly (over a second or longer), the flow rates will approach the accurate flow rates quite well within a few simulation time steps, and the overall error over time will be small. The advantage in model execution speed, however, is quite significant.

When modeling the loops, it is important to consider what happens when valves close. The best way to structure the loop network is to ensure that a branch with a valve in it is part of a single loop only; then, when the valve is closed, the flow rate in that loop does not have to be solved for, it can simply be set to zero. This will then result in zero flow through the valve.

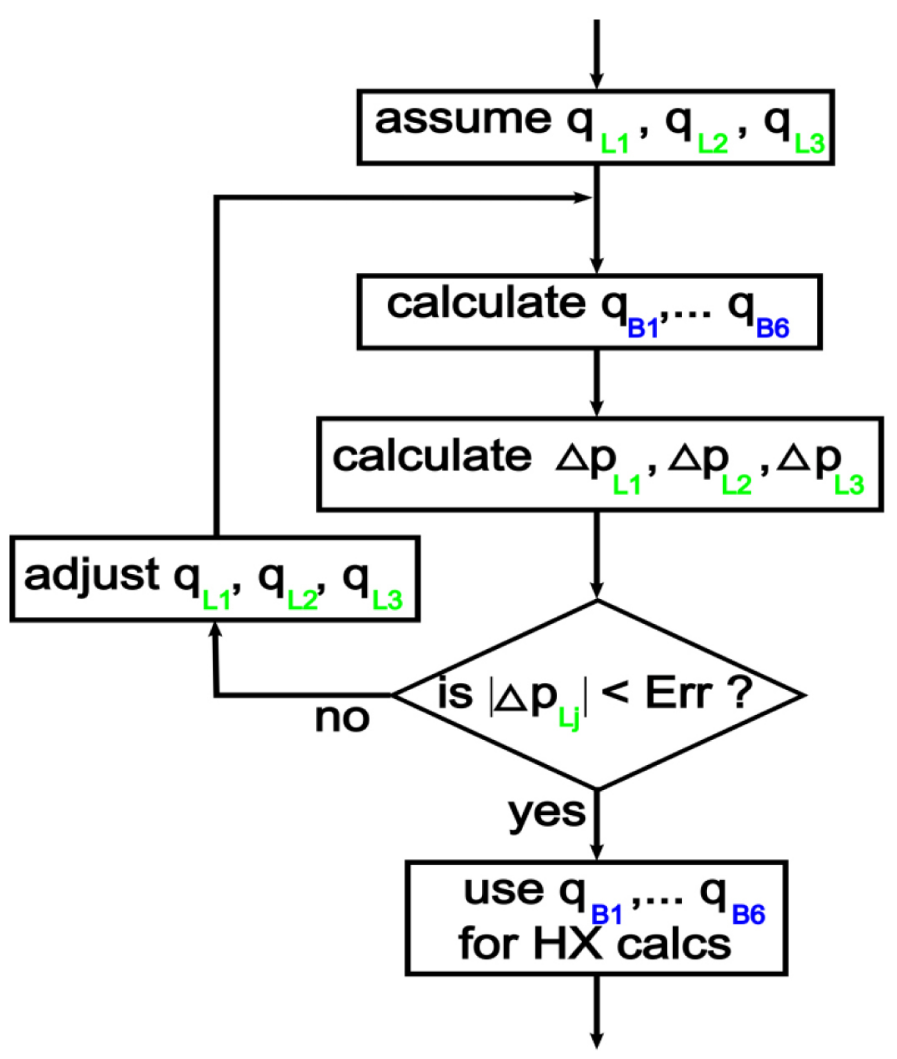

Figure 3. Coolant flow rate calculation flow chart

\section{Solving for Component Heat Transfer Rates in the Liquid Coolant-Based System}

As most heat transfer occurs in heat exchangers, the focus of this section is on the heat exchanger models. Other system components such as transport lines can be treated similarly. Two levels of detail are available for the heat exchanger models: the DistributedParameter and Mapped-Component models. The DistributedParameter model provides more detail and flexibility, while the Mapped-Component model provides faster execution time.

\section{Distributed-Parameter Models}

The Distributed-Parameter modelling method is described here using the example of a two-pass liquid coolant front end heat exchanger (FEHX). Figure 4 shows how this FEHX is represented in the model. The coolant and air flow across each of the flat tubes in a pass are assumed to be identical. Therefore, the flow for each flat tube in a pass can be calculated by dividing the total coolant mass flow rate by the number of flat tubes in that pass. The flat tubes in each pass can then be represented by one "liquid-coolant line with heat transfer" simulation block. Each FEHX pass can then 
be simplified in this manner as a single simulation block. The heat transfer in each pass is then the heat transfer rate in just one tube of the pass multiplied by the number of flat tubes in the pass. The total heat transfer rate for the FEHX is then the sum of the heat transfer rates for the two passes.

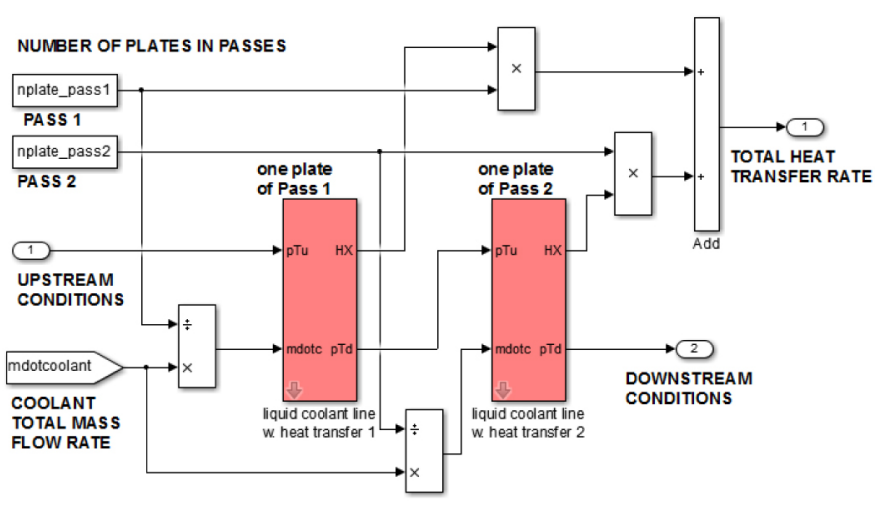

Figure 4. Distributed parameter model example for two-pass liquid coolant heat exchanger

In the "liquid coolant line with heat transfer" simulation block (shown in Figure 4), the tube is split up into a number of segments. In each segment, the coolant flow parameters, air flow parameters, wall temperature, and heat transfer rates are calculated individually with a "marching" scheme starting from the inflow boundary. Because this is a cross-flow heat exchanger, the air inflow parameters are the same on all segments. The coolant mass flow rate is also the same in all segments because steady-state flow rate of an incompressible liquid is assumed. The heat transfer rates, wall temperature, coolant-in temperature, and coolant-out temperature, however, will be different for each segment. The effectiveness-number of transfer units method is used for each segment [ $\underline{8}]$. The Chang correlation []ㅡ for the heat transfer coefficient on louvered fins is used on the air flow side, and the Dittus-Boelter correlation [ $[\underline{]}$ ] is used on the internal liquid coolant side. The coefficients used in these correlations are typically adjusted in a calibration process.

\section{Mapped-Component Models}

To increase the speed of model execution for long simulations, the Distributed-Parameter model can be used to generate a MappedComponent model. The process is similar to the one applied for generating the Mapped-Component $\mathrm{A} / \mathrm{C}$ system model using the Quasi-Transient A/C system simulation model [7]. The steps of this mapping process are as follows. First, create a DistributedParameter model of the heat exchanger with sufficient detail. Next, calibrate this model with available experimental data for a reasonably large set of measurement points. Then, map out the heat transfer rate over the entire operating range of the component. Implement this map into a lookup table in the thermal management system simulation model so that the heat exchange rate can be determined for any air and coolant inflow conditions over the entire operating range. Finally, use the inflow conditions and the heat exchange rate to determine the air and coolant outflow conditions for the heat exchanger.

\section{Application of Model for Combined Fluid Loops, Integrated Thermal Management}

NREL's combined fluid loop (CFL) EDV thermal management system test bench, shown as a schematic in Figure 5, was selected for the validation and demonstration of the modeling method. This test bench allows for testing a wide range of advanced $\mathrm{A} / \mathrm{C}$, heat pump, and cooling loop configurations $[\underline{10}]$.

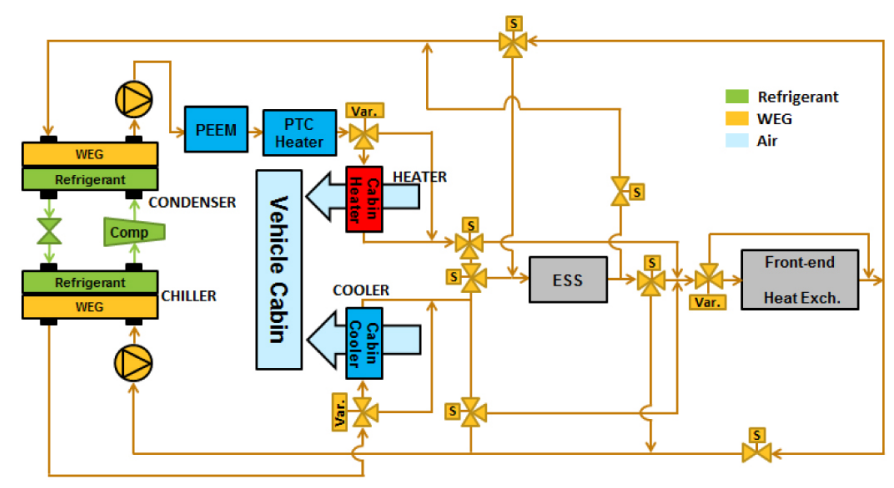

Figure 5. Schematic of NREL's CFL EDV thermal management system

In this system, a refrigerant circuit operating on the vapor compression cycle is used as both an $\mathrm{A} / \mathrm{C}$ unit (providing cooling) and as a heat pump (providing heating). The refrigerant loop heat exchange with the liquid coolant is in the chiller and in the condenser. The liquid coolant is used to provide cooling and heating to the cabin. "PEEM" in Figure 5 stands for the power electronics and electric motor, and "ESS" stands for energy storage system (battery pack). With proper setting of the valves, various modes of operation can be achieved. For example, the system can be reduced to heating (Figure 6) and cooling (Figure 7). Passive cooling and heating are also available when the refrigerant compressor is not operating and there is no refrigerant flow through the system. Further details of this system are described in a separate report [10].

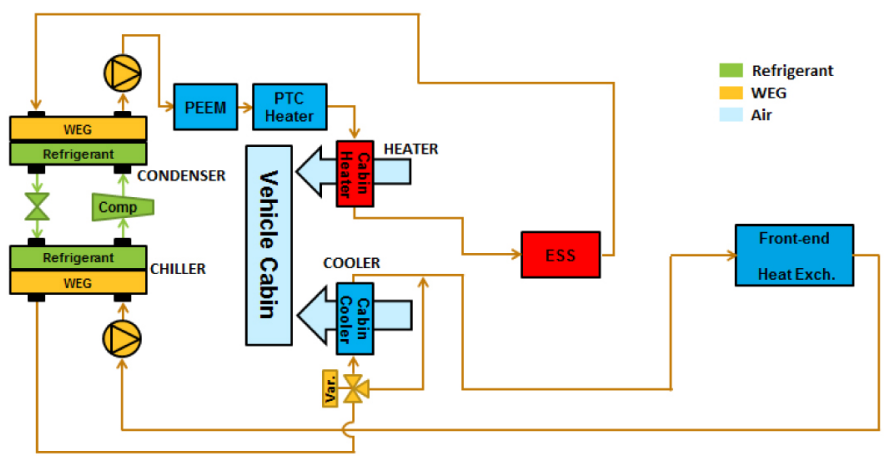

Figure 6. CFL heat pump mode

The developed simulation model can be used to predict processes in the experimental system for any combination of valve settings. The way the coolant loops were selected is shown in Figure 8. 


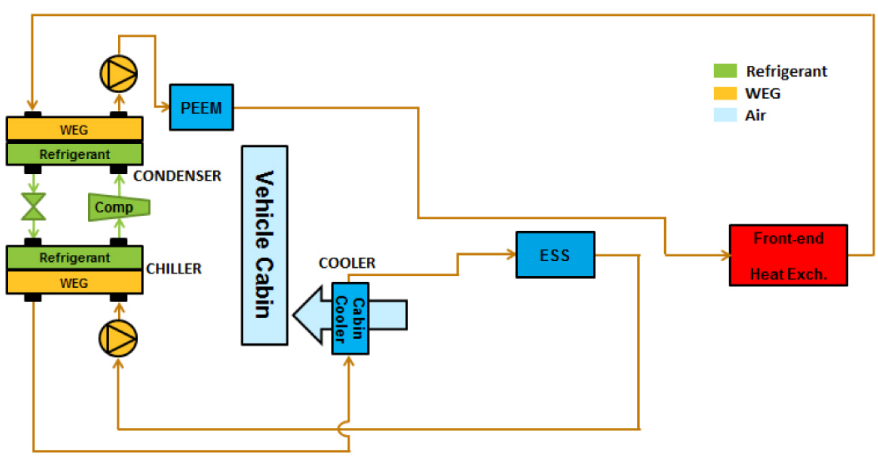

Figure 7. CFL A/C mode

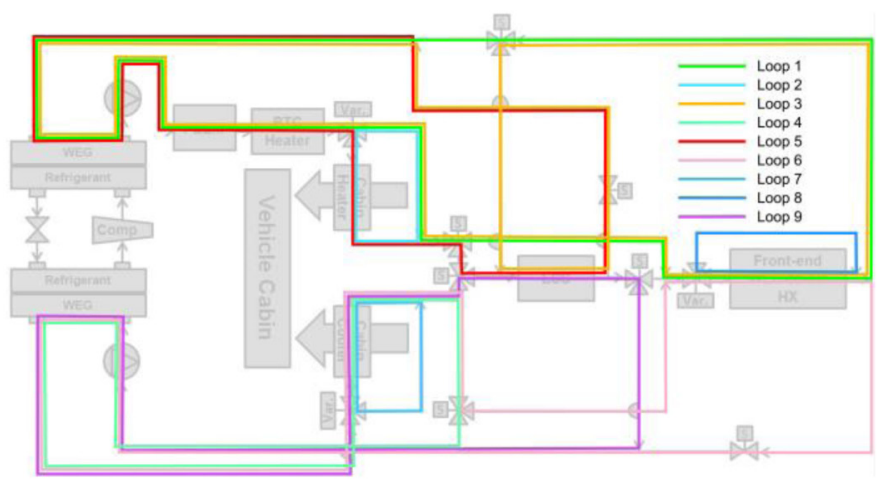

Figure 8. Set of coolant loops that allows modeling of all possible system operating modes

\section{Component Model Calibration}

Component information was provided by Delphi Automotive Systems, and measurements for ten different system operating conditions were conducted at NREL [10]. Component models were built, and a calibration process for all heat exchangers was carried out using these data. For calibration of the refrigerant-based heat exchangers, that is, the chiller and the condenser, the appropriate component models from the Quasi-Transient A/C system sub-model were used. As an example, the result of the calibration for the chiller is shown in Figure 9.
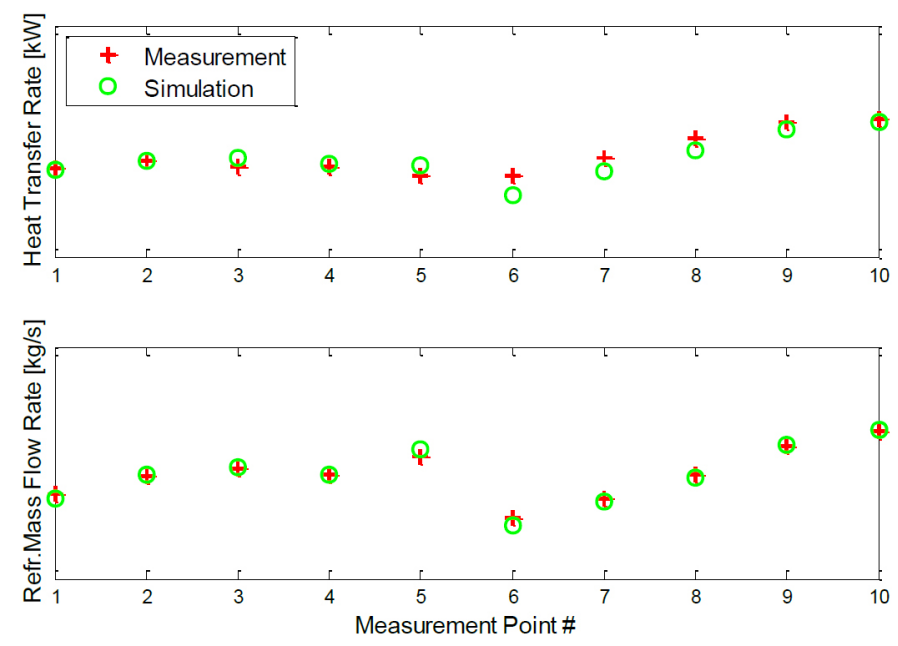

Figure 9. Calibration results for the chiller model using component data

Distributed-Parameter component models of the heat exchangers were created and calibrated on all three liquid coolant-based heat exchangers in the system. As an example, the results of this calibration for the FEHX are shown in Figure 10. Note the FEHX actually picks up heat when the system is in heat pump mode, which is why heat transfer rate takes on both negative and positive signs.

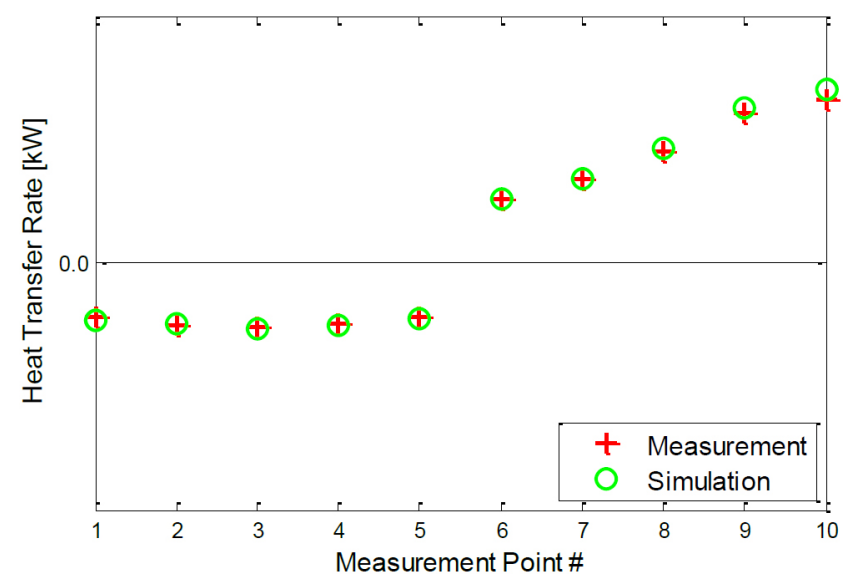

Figure 10. Heat transfer calibration for the FEHX. (Positive heat transfer means heat is transferred out of coolant)

The calibrated Distributed-Parameter models of the liquid coolantbased heat exchangers in the system were then used to generate maps of heat exchanger performance. These four-dimensional maps use the inlet liquid coolant mass flow rate and temperature and the air mass flow rate and temperature as the input parameters. Instead of using heat transfer rate as the output parameter, effectiveness was used to improve the interpolation between and possible extrapolation beyond the grid points. These lookup tables were then used in the Mapped-Component model, as shown in Figure 11, for the example of the FEHX.

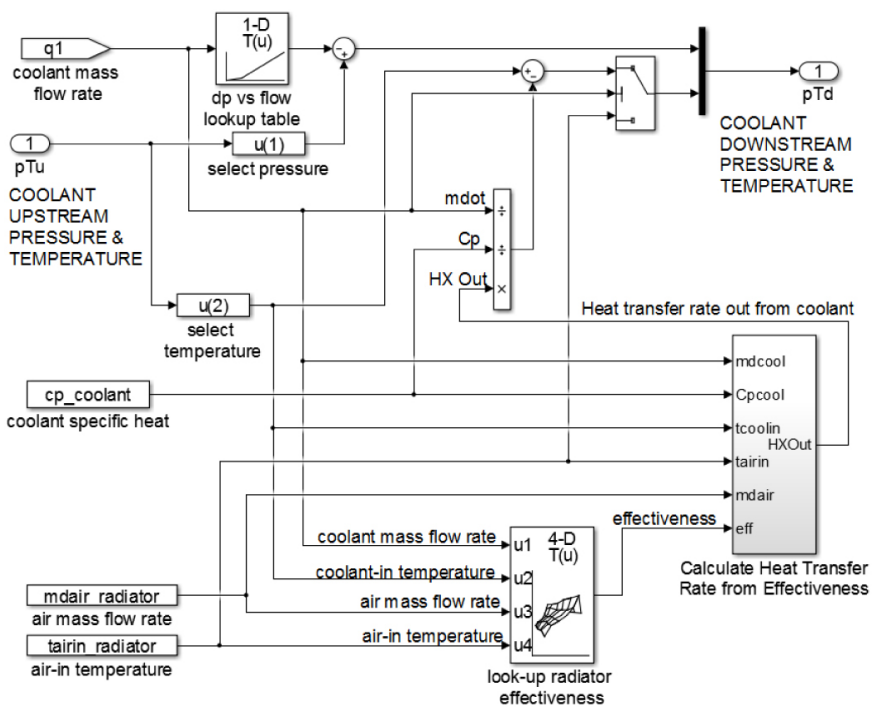

Figure 11. FEHX Mapped-Component model (simplified)

\section{Validation with Data for Full Combined Fluid Loop System}

Steady-state measured data for the CFL system in heating and cooling modes (Figure 6 and Figure 7) were available from NREL's experimental test bench [10]. The test bench instruments were calibrated and the uncertainties were propagated in accordance with American Society of Mechanical Engineers standards [11]. The ambient temperature of the system was swept between $-2^{\circ} \mathrm{C}$ and $+43^{\circ} \mathrm{C}$. A total of five points were below an ambient of $20^{\circ} \mathrm{C}$, for which the heat pump 
mode was used. Five points were above the $20^{\circ} \mathrm{C}$ ambient, for which the $\mathrm{A} / \mathrm{C}$ mode was used. The simulated vs. measured data were plotted as a function of the ambient temperature. The maximum $95 \%$ confidence interval uncertainty error bands are also shown on the plots except for the WEG temperatures and refrigerant pressures. The predicted and measured refrigerant flow rates are shown in Figure 12. Note that for the heat pump mode points, the temperature was outside the refrigerant mass flow meter's operating range resulting in inaccurate measurements. To provide more accurate data for the heat pump mode, the refrigerant flow rates were back calculated from the coolant side heat exchange rates and the refrigerant in- and out enthalpies. The capacities of the condenser and chiller, that is, the refrigerant-to-liquid coolant heat exchangers, are shown in Figure 13. The root mean square (RMS) error between simulation and measurement for these capacities is $4.3 \%$. Note the drop in condenser and chiller capacities in the heat pump mode operating points with $8^{\circ} \mathrm{C}$ ambient and above. The reason for this behavior is that in these points the system is limited by a $50^{\circ} \mathrm{C}$ cabin heater air-out temperature target. As ambient temperature is increasing, less cabin heater capacity is needed to meet this target, requiring reduced compressor speed. This also reduces the capacity on all the other heat exchangers in the system. The capacities of the liquid coolant heat exchangers are shown in Figure 14. Note that the actual heat transfer rate on the FEHX changes sign when the operating mode is switched from heating to cooling. When the heat pump mode is used, the FEHX accepts heat from the ambient air, and when the $\mathrm{A} / \mathrm{C}$ mode is used, the FEHX rejects heat to the ambient air. The RMS of errors between simulation and measurement for the heat exchange rates in the liquid coolant heat exchangers is $3.6 \%$. The coolant temperatures just upstream of the heat exchangers are shown in Figure 15. The maximum $95 \%$ confidence interval uncertainty for these measurements was 0.32 $\mathrm{K}$. The RMS of the errors between measurement and simulation is 1.20 $\mathrm{K}$. Finally, the system maximum and minimum refrigerant pressures, measured and simulated, are shown in Figure 16. The maximum 95\% confidence interval uncertainty for these measurements was $7 \mathrm{kPa}$ and $20 \mathrm{kPa}$ for the minimum and maximum pressures, respectively. The RMS value of the errors between simulation and measurement is $4.3 \%$. These RMS values of the simulation vs. measurement for all compared system variables indicate a good match between simulation and measurement. Furthermore, the simulated values are within the $95 \%$ measurement uncertainty band for $96 \%$ of the measurement points shown in Figures 12, 13, 14.

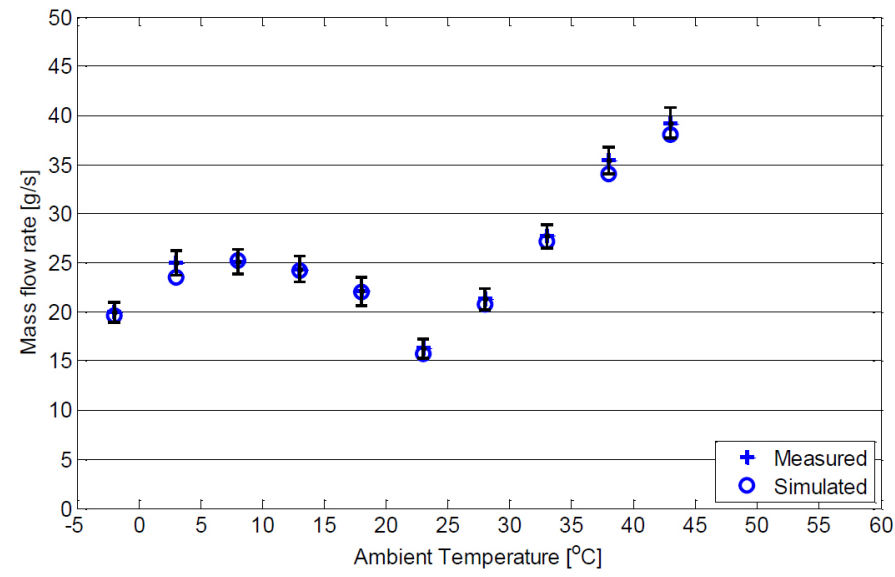

Figure 12. Refrigerant mass flow rates
The computational resource used to generate all results in this paper was a Dell Precision M4800 laptop computer with a $2.70 \mathrm{GHz}$ Intel $\AA$ Core $^{\text {TM }}$ 17-4800MQ processor and 8.00GB RAM and 64-Bit

Windows operating system. On this computer, the model runs at about $1 / 3^{\text {rd }}$ real-time speed. The ease of use of the model is difficult to quantify. 'User friendliness' of the model is best described as similar to the user friendliness of other Simulink models of similar complexity. Initialization and post-processing Simulink ' $m$ ' files have been developed. The great flexibility of the MATLAB/Simulink modeling environment allows the user to create / edit other pre- and post-processing files with relative ease.

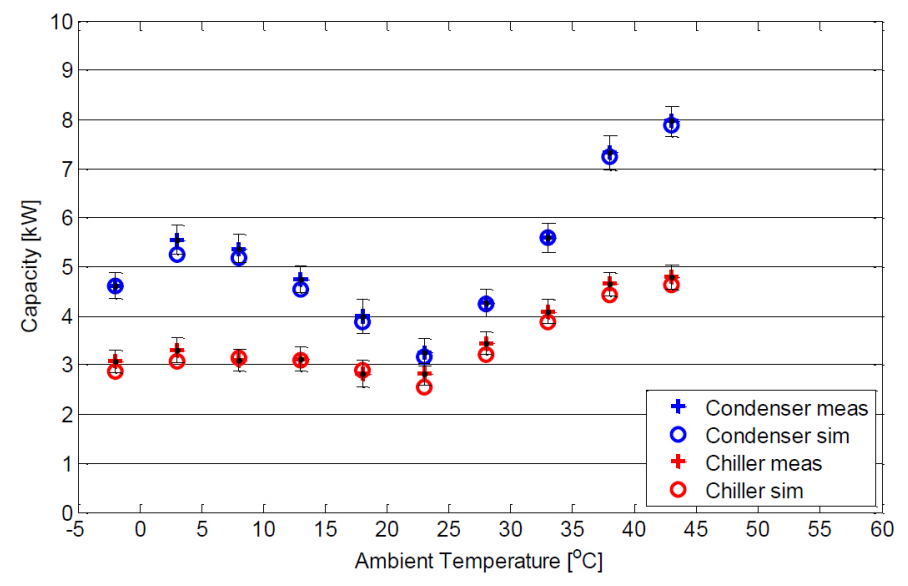

Figure 13. Capacities of chiller and condenser

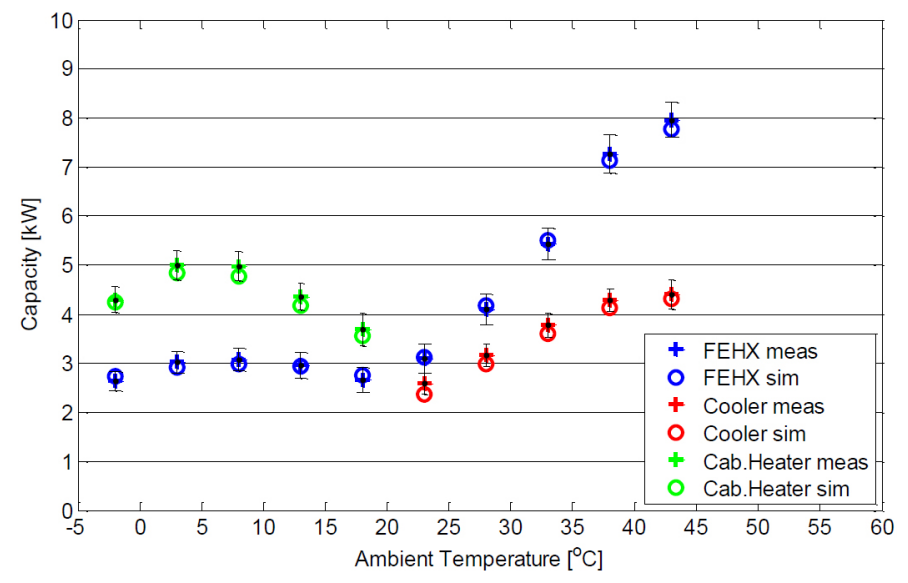

Figure 14. Capacities of liquid coolant-based heat exchangers

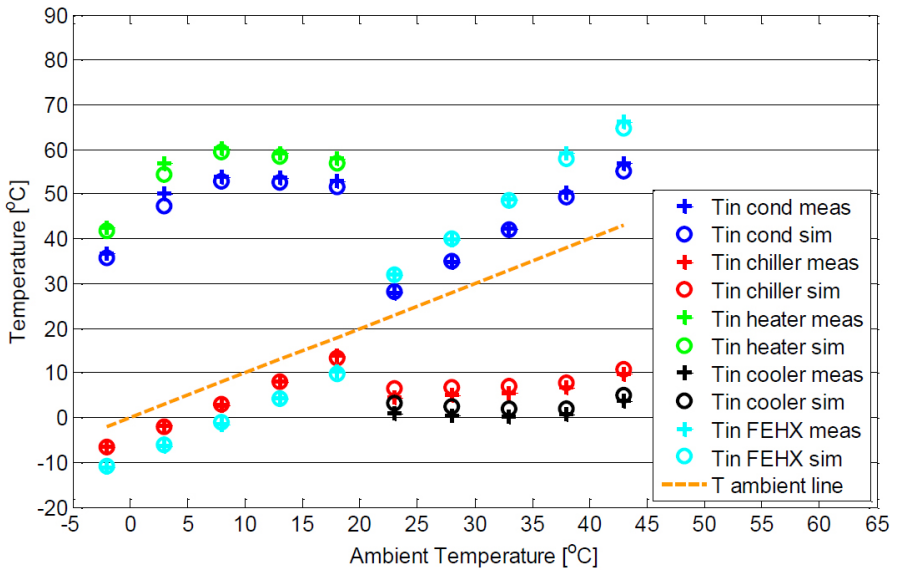

Figure 15. Coolant temperatures just upstream of heat exchangers 


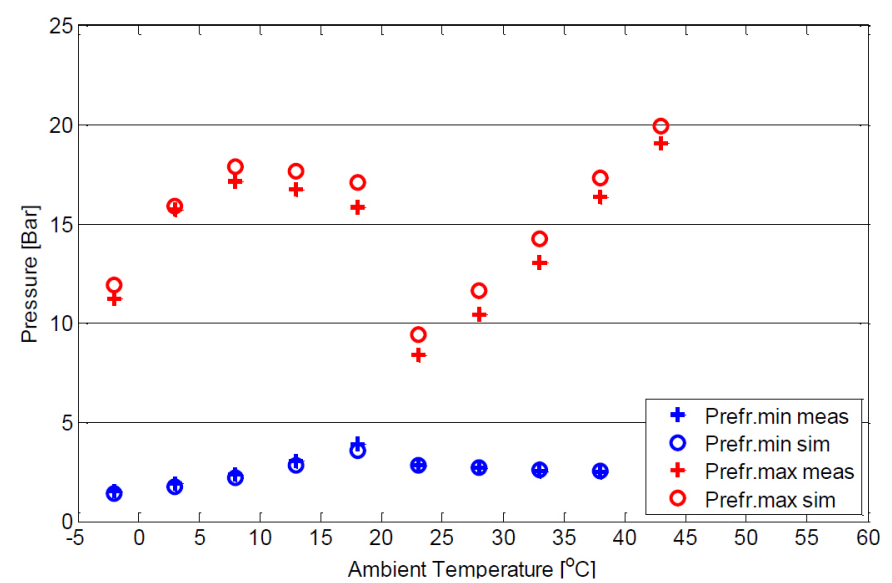

Figure 16. Refrigerant maximum and minimum pressures (compressor outlet and compression suction volume, respectively)

\section{Example Study: Determine Optimal Location of the PEEM}

The validated model can be used for various studies that help determine which operating mode is the best under different driving and ambient conditions. The model is also useful for exploring the advantages and disadvantages of system layouts different from that shown in Figure 5. For this study, the model was used to investigate the optimal location of the power electronics and electric motor (PEEM) module in the system. For the A/C mode (Figure 7), the optimal placement seems to be obvious: the PEEM needs to be in the condenser coolant circuit, where it is cooled by the coolant coming out of the condenser. Putting it in the chiller side coolant circuit would reduce the effectiveness of cabin cooling. On the other hand, for the heat pump mode (Figure 6), there is an argument to place the PEEM in the chiller side coolant circuit just upstream of the chiller. In this case, waste heat from the PEEM would not be directly available for heating. When the PEEM is in the chiller circuit, however, the coolant picks up heat in the PEEM and enters the chiller at a higher temperature. The heat pump coefficient of performance (COP) may be higher when the suction temperature is higher. Heating capacity can possibly be shifted from the positive temperature coefficient (PTC) heater to the heat pump. The loss of PEEM waste heat for cabin heating purposes could be more than compensated for by the higher COP of the heat pump. In that case, a system in which the PEEM can be switched between the two locations may be worth the extra cost of additional valves, coolant lines, and controls. It is useful to define the thermal system operating power (TSOP) as the total of the PTC heater and compressor power (blower and fan powers are neglected). Then the question can be stated as which PEEM location provides the lowest TSOP for cold start at various ambient temperatures for the same heating capacity. Simulation is well suited to answer this question.

\section{Setting Up the Model and Mapping Space}

Starting from a cold-soaked vehicle is the case where shortfall in heating capacity provided by the heat pump is the largest and PTC heat is most likely needed. Therefore, a cold start of the system was selected for evaluation of the PEEM placement. The comparison is done at a state just after the initial fastest transients in the refrigerant and coolant circuits have already settled out, which occurs within the first minute of operation. The system is then in a quasi-steady state in which various system variables are slowly changing as the vehicle is heating up. Such a quasi-steady state was approximated with true steady-state problem setup in which the cabin pressure, temperature and humidity were kept constant at ambient conditions by setting the cabin volume to a very large value. Then all cabin heat transfer pathways, including solar load, cabin shell convective heat transfer to the ambient air, and heat transfer from cabin interior to cabin air, had no effect on TSOP because the cabin heater operated at a constant air temperature. The cabin recirculation rate was set to $100 \%$, but that did not matter as the cabin conditions stayed the same as the ambient conditions.

The remaining system operating parameters that affect performance include the FEHX fan air flow rate, blower air flow rate, required cabin heating capacity, ESS temperature, ESS heat generation rate, PEEM heat transfer rate, and ambient temperature. The combined compressor and PTC powers (TSOP) can be evaluated on this seven-dimensional map; however, it made sense to reduce the scope of the problem with reasonable simplifications.

The FEHX fan and blower air volumetric flow rates were kept constant 1320 and 210 SCFM for this study. The cabin heater exit air temperature was controlled to $50^{\circ} \mathrm{C}$ to achieve fast initial cabin heating. From this requirement and from the cabin temperature and the blower air flow rate, the required heating capacity was set. Therefore, the heating capacity did not have to be included as a varied mapping parameter. Because the heat pump COP is expected to be greater than 1 , as much of the heating demand as possible should be provided with the heat pump. Therefore, the compressor speed was first varied up to its maximum 6,200 RPM to meet the cabin heater air-out temperature target. Any shortfall in cabin heater air-out temperature was then made up with the PTC heater. In the temperature range investigated, the PTC heater was always needed to supplement the heat pump. If the COP ever dropped below 1.0, the TSOP would simply be set to the required heating capacity, accounting for all the heat coming from the PTC heater with a COP of 1.0. For all the simulations, however, the COP stayed above 1.0.

ESS heating in a cold start is important so that an efficient operating temperature can be reached quickly. Therefore, it is assumed that the coolant can be partially bypassed around the ESS to achieve various levels of heating rate, regardless of the ESS temperature. The controls related to how much coolant heat energy to use for heating the ESS can be quite complex, as the control algorithm has to make a compromise between several competing factors. These factors include driving range, battery life, and cabin heating capacity. Instead of trying to implement such an ESS heating algorithm, it made sense to use the ESS heat transfer rate, and not the ESS heat generation rate, as a variable parameter for the study.

After these considerations, the ambient temperature, the PEEM heat transfer rate and the ESS heat transfer rate remained as the parameters over which the combined compressor and PTC power had to be mapped.

Performance of the compressor is an important input to the models for this study. Unfortunately, limited data, which excluded the lowest temperatures, was available. Only four operating points were available for the compressor in which the RPM was at the maximum 6200 . The pressure ratio range within these four points was 6.60 to 
8.24 , which is a rather narrow range. Within this range, the volumetric, isentropic, and overall efficiencies were nearly constant, and there was no noticeable trend as a function of the pressure ratio. Therefore, constant values for these three efficiencies were set to the average of the four points. Finally, in the study, the water/ethylene glycol (WEG) coolant flow rates were set to $0.24 \mathrm{~kg} / \mathrm{sec}$ for both system configurations, for both the condenser and chiller loops, and for all conditions.

The system configuration of interest allows the placement of the PEEM in the condenser coolant loop or the chiller coolant loop with the activation/deactivation of various valves. The PEEM in the chiller loop system would be different from the current NREL test bench, but the validated model can easily be modified for this PEEM location study. Instead of modifying the loop topology and thereby allowing the same model to be used for both cases, it was simpler to have two models. In one model, the PEEM is in the condenser loop downstream of the condenser and coolant pump. In the other model, the PEEM is in the chiller coolant loop just upstream of the chiller. Otherwise, these two model versions are identical, and they are set up for the heat pump mode with the proper activation of coolant loops.

\section{Comparison for One Operating Point}

Before mapping across the complete variable range, the two system options were compared for one operating point, defined by a $-7^{\circ} \mathrm{C}$ ambient temperature, PEEM-to-coolant heat transfer rate of $1.25 \mathrm{~kW}$, and coolant-to-EES heat transfer rate of $1.5 \mathrm{~kW}$. The coolant temperature was plotted along the coolant loop in the flow direction. The plots for the PEEM in the condenser loops are shown in Figures $\underline{17}$ and $\underline{18}$ for the condenser loop and the chiller loop, respectively.

The comparable plots for the PEEM in the chiller loop are shown in Figures 19 and 20 .

Note that in these plots, coolant temperature changes through the heat exchangers are proportional to heat exchange rates because the coolant flow rates are the same. Coolant temperature changes over valves and coolant lines that are not dedicated heat exchangers are marked with black lines. As can be seen in Figures 17 and 19, the overall temperature distribution in the condenser loop is very similar for the two cases. Because all cabin air heating takes place across the cabin heater, the cabin heating capacity is set by the ambient temperature. As the coolant flow rate and the air inlet flow variables into the heater core are the same, the coolant inlet temperatures to the cabin heater have to be the same for the two configurations to achieve the same heating capacity. The other system component over which the coolant temperature drops is the ESS, and because the coolant mass flow rate and ESS heat transfer rate are the same between the two cases, the temperature drop over the ESS must be the same too. Then, the total temperature drop in the condenser coolant loop also has to be the same between the two configurations. Furthermore, this means the total temperature rise in the condenser loop also has to be the same between the two configurations. The key difference in temperature distribution is that when the PEEM is in the condenser loop, part of the temperature rise takes place over the PEEM, which reduces the heat transfer rates in the condenser and the PTC heater compared to when the PEEM is in the chiller loop.

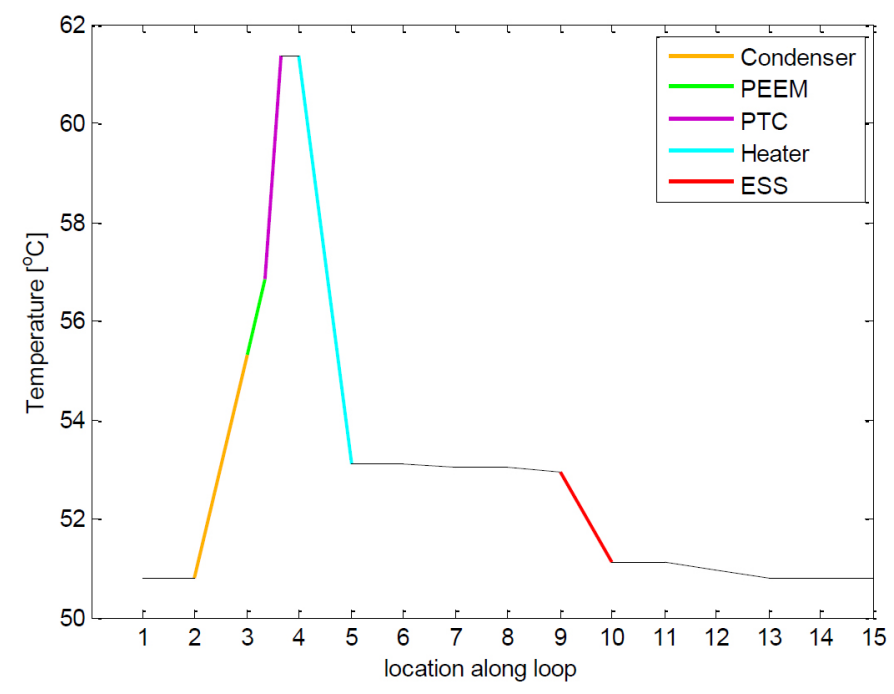

Figure 17. Coolant temperature along the condenser loop for the case of PEEM in the condenser loop for the $-7^{\circ} \mathrm{C}$ ambient case

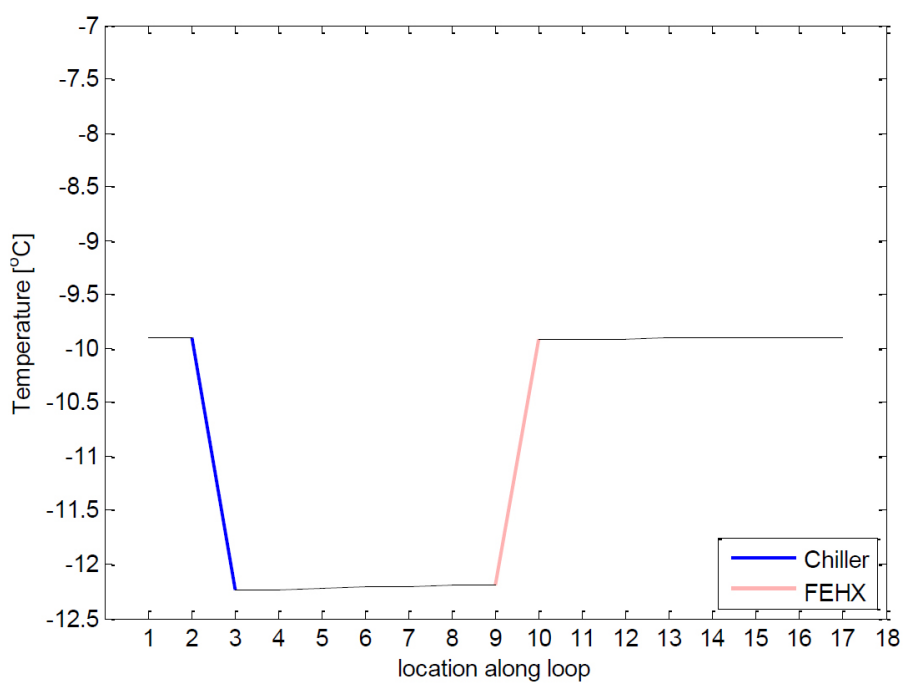

Figure 18. Coolant temperature along the chiller loop for the case of PEEM in the condenser loop for the $-7^{\circ} \mathrm{C}$ ambient case

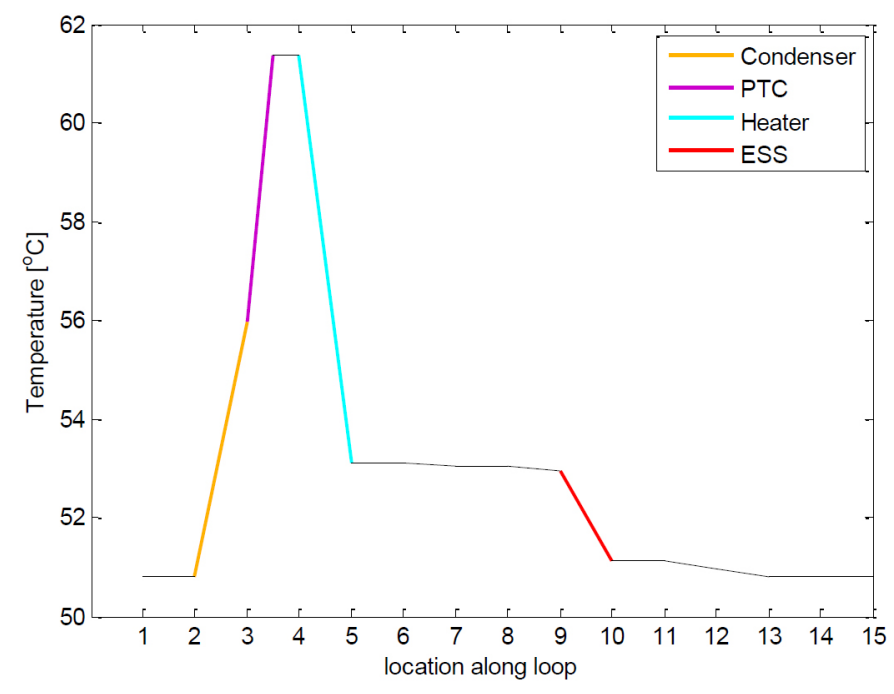

Figure 19. Coolant temperature along the condenser loop for the case of PEEM in the chiller loop for the $-7^{\circ} \mathrm{C}$ ambient case 


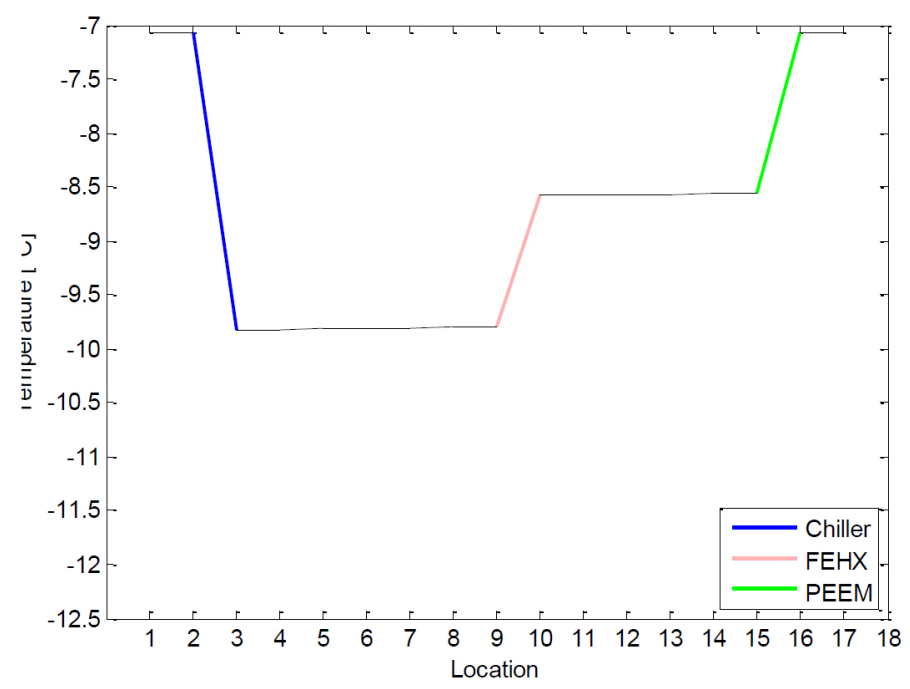

Figure 20. Coolant temperature along the chiller loop for the case of PEEM in the chiller loop for the $-7^{\circ} \mathrm{C}$ ambient case

Comparing the chiller loop temperature distributions in Figures 18 and 20, the temperatures are higher when the PEEM is in the chiller loop. This is expected as the PEEM is an additional heat source available to heat the coolant. A second effect is that the coolant temperature rise in the FEXH (and therefore heat transfer rate in the FEHX) is about half when PEEM is in the chiller loop.

\section{Performance Mapping}

In mapping the system performance, the PEEM heat generation rate values covered the range between a low-power city driving average $(0.5 \mathrm{~kW})$ and a high-power highway driving average $(2.75 \mathrm{~kW})$ with $0.75 \mathrm{~kW}$ steps. For the ESS heat transfer rate, values of $0.75,1.5$, and $2.25 \mathrm{~kW}$ were used. The ambient temperature was varied between $-22^{\circ} \mathrm{C}$ and $8^{\circ} \mathrm{C}$ in $5^{\circ} \mathrm{C}$ increments. These grid point selections for PEEM, ESS, and ambient temperature gave a total of 84 operating points to evaluate. The mapping was done for both cases of PEEM location in the system with the two separate simulation models, respectively. The models were run to steady state conditions. Some relevant results for the PEEM to coolant heat transfer rate of $1.25 \mathrm{~kW}$ and coolant to ESS heat transfer rate of $1.5 \mathrm{~kW}$ case are shown in Figure 21. All the plots in the figure show system variables as functions of the ambient temperature for both PEEM location cases. The top plot shows the cabin heater capacities and the PTC capacities. Because the PTC heats the coolant and not the air directly, all air heating takes place on the cabin heater. Regardless of the PEEM location, the cabin heater capacity should be the same for both PEEM location cases, and they are. However, the PTC heater capacities differ because when the PEEM is in the condenser loop, there is extra heating from the PEEM, which allows a reduction in the required heat input from the PTC. This was also shown when comparing the coolant temperatures in Figures 17 and $\underline{19}$. The second and third plots in Figure 21 are the maximum (compressor outlet) and the minimum (compressor inlet) refrigerant pressures in the system, respectively. While the outlet pressures are nearly the same, the suction pressure is significantly higher when the PEEM is in the chiller loop. The fourth plot shows the refrigerant mass flow rate. With the higher pressure and same superheat in the suction volume, the suction volume refrigerant density is higher when the PEEM is in the chiller loop, and the refrigerant mass flow rate is higher. With the maximum refrigerant pressures nearly the same, but the minimum refrigerant temperature higher for the PEEM in the chiller loop case, the compressor pressure ratio is lower, resulting in better heat pump COP as shown in the fifth plot of Figure 21. Note that the COP difference is not very significant. Despite the improved COP, the compressor power is still higher for this case due to the higher refrigerant mass flow rate, as shown in the sixth plot of Figure 21. Because the PTC heater power was also higher for the PEEM in the chiller loop case, the total of the PTC heater power and compressor power, the TSOP, is also higher when the PEEM is in the chiller loop.
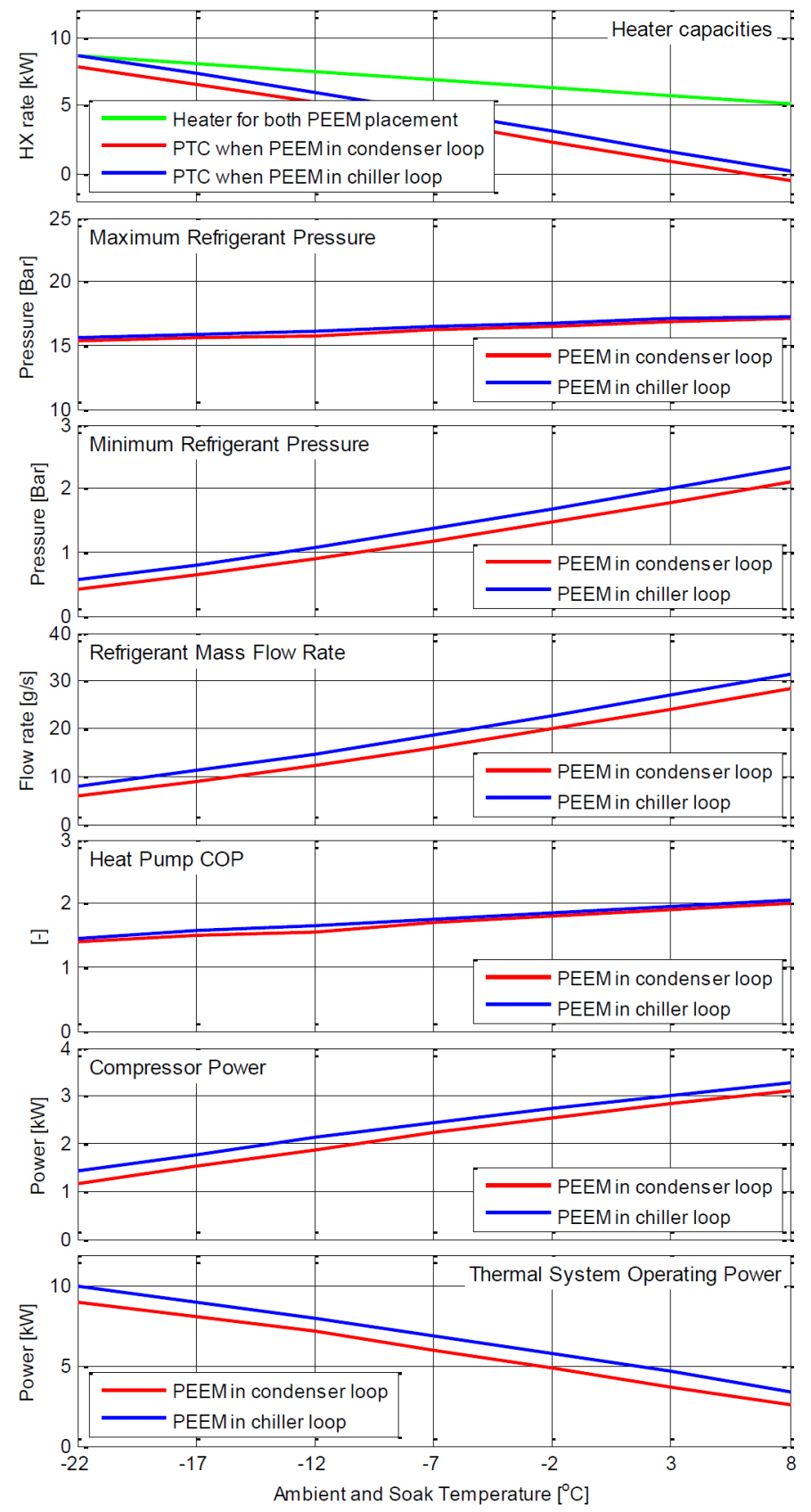

Figure 21. Various system variables as function of ambient temperature for PEEM and EES heat transfer rates of $1.25 \mathrm{~kW}$ and $1.5 \mathrm{~kW}$ respectively

The same trends were found for all other combinations of these heat transfer rates. The thermal system with the PEEM in the chiller loop always used more total power than the system with the PEEM in the condenser loop. Finally, note that in the investigated system the PTC heats the coolant not the air directly. A significant advantage of the PTC heater heating the air directly is the quicker response of the vent air-out 
temperature as the coolant does not need to be heated up before the PTC heater's effect on the air temperature is fully in place. Similar studies were carried out on the PTC used for direct heating of the air in series with the heater, and the same conclusion was found: the PEEM in the condenser loop used less thermal system operating power.

\section{Conclusions}

NREL's MATLAB/Simulink A/C modeling toolset, CoolSim, was extended to incorporate simulation of liquid coolant subsystems. This enables simulation of an entire EV thermal management system. This new modeling methodology is especially useful for the simulation of thermal management systems where the refrigerant- and liquid coolant-based thermal sub-systems can be highly influenced by one another, such as in advanced hybrid and electric vehicles.

These new simulation capabilities were applied to an advanced combined cooling loop EV thermal management system concept. Predicted system parameters for ten steady state-system operating points were compared against measurement data obtained on an experimental test bench, and good agreement was found between them. The RMS error between predicted and measured coolant temperatures was found to be $1.20 \mathrm{~K}$, and the RMS error between predicted and measured heat exchanger capacities across all heat exchangers and all operating points was found to be $3.9 \%$.

The simulation model was applied to study whether placing the PEEM in the condenser coolant loop or the chiller coolant loop is more beneficial. Placing the PEEM in the condenser coolant loop results in lower thermal system operating power for the particular system studied.

As demonstrated in this work, the new thermal system simulation modelling method can be effectively used to evaluate various options that may be available for configurations of EDV thermal systems. Similarly, it should also be useful for evaluating the various operating modes that may be possible in a given complex thermal management system, thereby aiding the pairing of the most effective and efficient operating mode to any given driving and ambient conditions.

\section{References}

1. Rugh, J., Hovland, V., and Andersen, S. O., "Significant Fuel Savings and Emission Reductions by Improving Vehicle Air Conditioning," Earth Technologies Forum/Mobile Air Conditioning Summit, 2004

2. Francfort, J., and Murphy, T., (2007). Ch. V. "Operational and Fleet Testing, A. Hybrid Electric Vehicle Testing,” Advanced Vehicle Technology Analysis and Evaluation Activities, FY2007 Annual Report. Vehicle Technologies Program, U.S. Department of Energy. p. 145.

3. Umezu, K., and Noyama, H. "Air-Conditioning System for Electric Vehicles (i-MiEV)," presented at SAE Automotive Refrigerant and System Efficiency Symposium, 2010.

4. Rask, E., Lohse-Busch, H., Duoba, M., and Stutenberg, K., "Ford Focus BEV In-depth (Level 2) Testing and Analysis," DOE Annual Merit Review Presentation. 2014

5. Computer Software "Autonomie," www.autonomie.net, January 19,2015
6. Kiss, T., Chaney, L., and Meyer, J., "A New Automotive Air Conditioning System Simulation Tool Developed in MATLAB/ Simulink," SAE Int. J. Passeng. Cars - Mech. Syst. 6(2):826840, 2013, doi:10.4271/2013-01-0850.

7. Kiss, T. and Lustbader, J., "Comparison of the Accuracy and Speed of Transient Mobile A/C System Simulation Models," SAE Int. J. Passeng. Cars - Mech. Syst. 7(2):739-754, 2014, doi:10.4271/2014-01-0669.

8. Incropera, F.P., and DeWitt, D.P., Fundamentals of Heat and Mass Transfer, $2^{\text {nd }}$ edition, 1985, John Wiley and Sons, Inc.

9. Chang, Y.J., and Wang, C.C., "A Generalized Heat Transfer Correlation for Louver Fin Geometry," Int. J. Heat Mass Transfer 40(3):533-544, 1997. doi:10.1016/00179310(96)00116-0.

10. Leighton, D., "Combined Fluid Loop Thermal Management for Electric Drive Vehicle Range Improvement," SAE Technical Paper 2015-01-1709, 2015, In Press.

11. Dieck, R.H., Steele, W.G., and Osolsobe, G., Test Uncertainty. ASME PTC 19.1-2005. New York, NY: American Society of Mechanical Engineers. 2005.

\section{Contact Information}

Tibor Kiss

tibor.kiss@nrel.gov

Jason Lustbader

jason.lustbader@nrel.gov

\section{Acknowledgments}

The authors would like to thank

1. Delphi for component bench data

2. David Anderson and Lee Slezak, Technology Managers for the U.S. Department of Energy's Advanced Vehicle Technology Analysis and Evaluation for sponsoring this work.

\section{Definitions/Abbreviations}

\author{
${ }^{\circ} \mathbf{C}$ - degrees Celsius \\ A/C - air conditioning \\ CFL - combined fluid loop \\ COP - coefficient of performance \\ EDV - electric-drive vehicle \\ ESS - energy storage system (batteries) \\ EV - electric vehicle \\ FEHX - front end heat exchanger \\ K - Kelvin \\ NREL - National Renewable Energy Laboratory \\ PEEM - power electronics and electric motor \\ PTC - positive temperature coefficient \\ RMS - root mean square \\ TSOP - thermal system operating power \\ WEG - water-ethylene glycol coolant mix
}




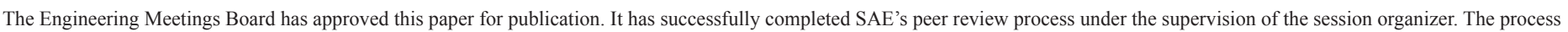
requires a minimum of three (3) reviews by industry experts.

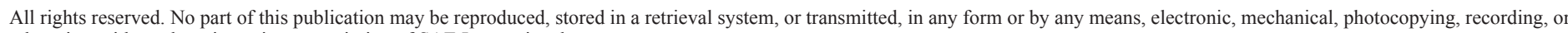
otherwise, without the prior written permission of SAE International.

Positions and opinions advanced in this paper are those of the author(s) and not necessarily those of SAE International. The author is solely responsible for the content of the paper.

ISSN 0148-7191

http://papers.sae.org/2015-01-1708 